\title{
Swift observations of GRB 050904: the most distant cosmic explosion ever observed
}

\author{
G. Cusumano ${ }^{1}$, V. Mangano ${ }^{1}$, G. Chincarini ${ }^{2,3}$, A. Panaitescu ${ }^{4}$, D. N. Burrows ${ }^{5}$, V. La Parola ${ }^{1}$, T. Sakamoto ${ }^{6,7}$, \\ S. Campana ${ }^{2}$, T. Mineo ${ }^{1}$, G. Tagliaferri ${ }^{2}$, L. Angelini ${ }^{6}$, S. D. Barthelmy ${ }^{6}$, A. P. Beardmore ${ }^{8}$, P. T. Boyd ${ }^{6}$, \\ L. R. Cominsky ${ }^{9}$, C. Gronwall ${ }^{5}$, E. E. Fenimore ${ }^{4}$, N. Gehrels ${ }^{6}$, P. Giommi ${ }^{10}$, M. Goad ${ }^{8}$, K. Hurley ${ }^{11}$, S. Immler ${ }^{6}$, \\ J. A. Kennea ${ }^{5}$, K. O. Mason ${ }^{12}$, F. Marshal ${ }^{6}$, P. Mészáros ${ }^{5,13}$, J. A. Nousek ${ }^{5}$, J. P. Osborne ${ }^{8}$, D. M. Palmer ${ }^{4}$, \\ P. W. A. Roming ${ }^{5}$, A. Wells ${ }^{8}$, N. E. White ${ }^{6}$, and B. Zhang ${ }^{14}$
}

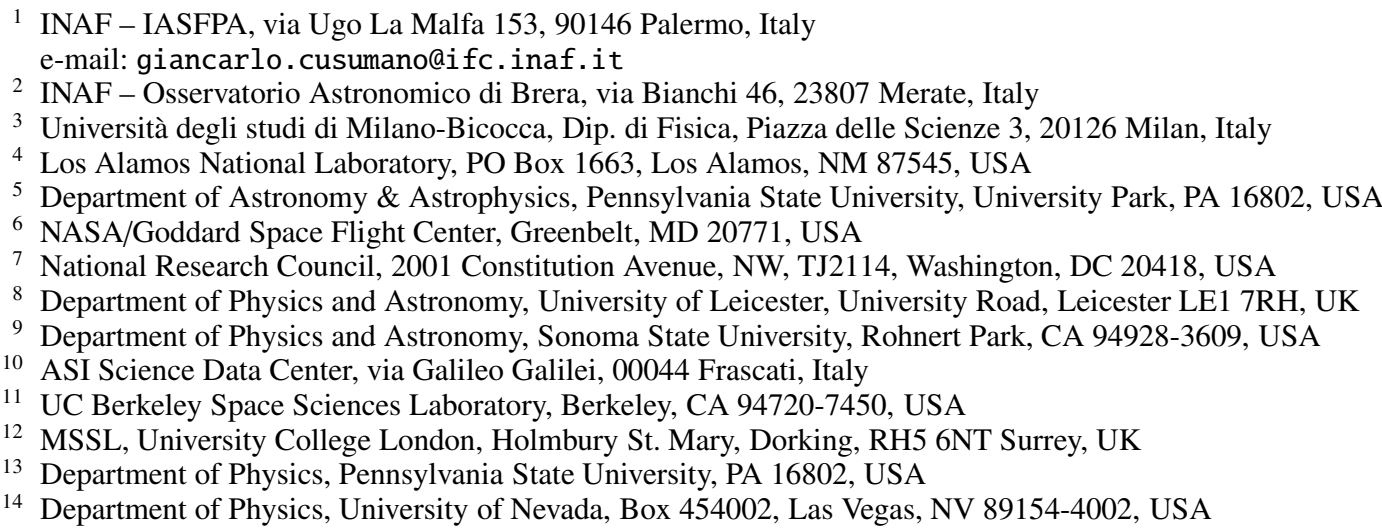

Received 9 March 2006 / Accepted 5 October 2006

\section{ABSTRACT}

\begin{abstract}
Context. Swift discovered the high redshift $(z=6.29)$ GRB 050904 with the Burst Alert Telescope (BAT) and began observing with its narrow field instruments $161 \mathrm{~s}$ after the burst onset. This gamma-ray burst is the most distant cosmic explosion ever observed. Because of its high redshift, the X-ray Telescope (XRT) and BAT simultaneous observations provide 4 orders of magnitude of spectral coverage $(0.2-150 \mathrm{keV} ; 1.4-1090 \mathrm{keV}$ in the source rest frame) at a very early source-frame time (22 s). The X-ray emission was monitored by the XRT up to 10 days after the burst.

Aims. We present the analysis of BAT and XRT observations of GRB 050904 and a complete description of its high energy phenomenology.

Methods. We performed time resolved spectral analysis and light curve modeling.

Results. GRB 050904 was a long, multi-peaked, bright GRB with strong variability during its entire evolution. The light curve observed by the XRT is characterized by the presence of a long flaring activity lasting up to $1-2 \mathrm{~h}$ after the burst onset in the burst rest frame, with no evidence of a smooth power-law decay following the prompt emission as seen in other GRBs. However, the BAT tail extrapolated to the XRT band joins the XRT early light curve and the overall behavior resembles that of a very long GRB prompt. The spectral energy distribution softens with time, with the photon index decreasing from -1.2 during the BAT observation to -1.9 at the end of the XRT observation. The dips of the late X-ray flares may be consistent with an underlying X-ray emission arising from the forward shock and with the properties of the optical afterglow reported by Tagliaferri et al. (2005b, A\&A, 443, L1).

Conclusions. We interpret the BAT and XRT data as a single continuous observation of the prompt emission from a very long GRB. The peculiarities observed in GRB 050904 could be due to its origin within one of the first star-forming regions in the Universe; very low metallicities of the progenitor at these epochs may provide an explanation.
\end{abstract}

Key words. gamma rays: bursts - X-rays: bursts - gamma rays: observations - cosmology: early Universe

\section{Introduction}

Gamma-Ray Bursts are bright flashes of high energy photons that can last from about $10 \mathrm{~ms}$ to $10 \mathrm{~min}$. Their origin and nature puzzled the scientific community for about 25 years until 1997, when the first X-ray afterglow of long ( $>2$ s duration) bursts were detected (Costa et al. 1997), and the first optical (van Paradijs et al. 1997) and radio (Frail et al. 1997) counterparts were found. These measurements established that long
GRBs are typically at high redshift $(z \sim 1.6)$ and are in subluminous star-forming host galaxies (Bloom et al. 2002). They are likely produced in core-collapse explosions of a class of massive stars that give rise to highly relativistic jets (the collapsar model, MacFadyen et al. 2001). Internal inhomogeneities in the velocity field of the relativistic expanding flow lead to collisions between fast moving and slow moving fluid shells and to the formation of internal shock waves (Rees \& Mészáros 1994). These shocks are believed to produce the observed prompt emission 
in the form of irregularly shaped and spaced pulses of gammarays, each pulse corresponding to a distinct internal collision. The expansion of the jet outward into the circumburst medium is believed to give rise to "external" shocks, responsible for producing the smoothly fading afterglow emission seen in the X-ray, optical and radio bands (Mészáros \& Rees 1997).

The Swift (Gehrels et al. 2004) X-ray Telescope (XRT, Burrows et al. 2005b) is providing a growing number of unprecedented observations of the early stages of GRB afterglow in the $0.2-10 \mathrm{keV}$ X-ray band. The XRT rapid ( $\leq 2 \mathrm{~min}$ ) response to the Swift Burst Alert Telescope (BAT, Barthelmy et al. 2005a) triggers has already led to the discovery of rapid early X-ray declines followed by the smoother "standard" X-ray afterglow components (Tagliaferri et al. 2005a; Cusumano et al. 2006a; Barthelmy et al. 20005b; Vaughan et al. 2006a), dramatic flaring in the early X-ray light curves of short (Fox et al. 2005; Barthelmy et al. 2005c; Campana et al. 2006; Vaughan et al. 2006b) and long bursts (Burrows et al. 2005a; Romano et al. 2006; Falcone et al. 2006; Burrows et al. 2005c; Pagani et al. 2006; Morris et al. 2006) and simultaneous peaks at the end of the BAT observation $(15-350 \mathrm{keV})$ and at the beginning of the XRT observation $(0.2-10 \mathrm{keV})$ of some long bursts (GRB 050730, GRB 050820a, GRB 050822; see O'Brien et al. 2006). Thanks to its fast response, and precise source localization, about $3^{\prime}$ in BAT, $3^{\prime \prime}-5^{\prime \prime}$ in XRT and $0.3^{\prime \prime}$ in UVOT (Roming et al. 2006), Swift is able to quickly alert ground-based telescopes to locate the optical counterpart and get redshift measurements before the object becomes too faint.

GRB 050904 triggered the BAT on 2005 September 4 at 01:51:44 UT (Cummings et al. 2005). The burst was located on-board at $\mathrm{RA}_{\mathrm{J} 2000}=00^{\mathrm{h}} 54^{\mathrm{m}} 41^{\mathrm{s}}, \operatorname{Dec}_{\mathrm{J} 2000}=+14^{\circ} 08^{\prime} 17^{\prime \prime}$ with an uncertainty of $3^{\prime}$ radius ( $90 \%$ confidence level) and the spacecraft was quickly pointed towards it. The XRT on-board centroiding algorithm failed to detect the counterpart due to the presence of a hot CCD detector column. The burst was long and bright with duration $T_{90}=(225 \pm 10) \mathrm{s}$ and a $15-150 \mathrm{keV}$ fluence of $(5.4 \pm 0.2) \times 10^{-6} \mathrm{erg} \mathrm{cm}^{-2}$ (Sakamoto et al. 2005). UVOT did not detect the burst counterpart down to a 3 sigma upper limit of about $21 \mathrm{mag}$ in all its six filters (Cucchiara et al. 2005). The optical detection was first made by the robotic observatory TAROT that begun to observe GRB050904 only $81 \mathrm{~s}$ after the Swift trigger. A bright optical flare was detected during the prompt high-energy emission phase (Boër et al. 2006; Gendre et al. 2006).

Early photometry indicated a high redshift $(z>5$, Reichart 2005). A photometric redshift $z=6.1_{-0.12}^{+0.37}$ was measured by Tagliaferri et al. (2005b) and confirmed by a Subaru spectroscopic measurement of $6.29 \pm 0.01$ (Kawai et al. 2005). A break at $T_{\mathrm{b}}=2.6 \pm 1.0$ days was also found in the $J$-band light curve (Tagliaferri et al. 2005b).

Such a high redshift means that this explosion happened 12.8 billion years ago $^{1}$, corresponding to a time when the Universe was young ( $\leq 1 \mathrm{Gyr}$ ), close to the re-ionization era (Becker et al. 2001). This gave GRB 050904 the distinction of being the most distant cosmic explosion ever observed: the previous record for a GRB was 4.5 (Andersen et al. 2000), the most distant quasar known is at a redshift of 6.4 (Fan et al. 2003), and the most distant galaxy is at a redshift of $\sim 7$ (Kneib et al. 2004).

Here we present the analysis of the BAT and XRT observations of GRB 050904. The details on data reduction are in Sect. 2; temporal and spectral analysis results are reported in

1 We used standard cosmological parameters of $\Omega_{\mathrm{M}}=0.27, \Omega_{\Lambda}=$ $0.73, H_{0}=71 \mathrm{~km} \mathrm{~s}^{-1} \mathrm{Mpc}^{-1}$.
Sects. 3 and 4, respectively. In Sect. 5 we draw our conclusions. Our results have already been shortly summarized by Cusumano et al. (2006b), but this paper contains a complete description of the phenomenology of the GRB 050904. Compared to the Watson et al. (2005) paper, that already presented the XRT data analysis, we add: (i) the detailed BAT data analysis; (ii) complete and correct BAT and XRT light curves; (iii) time resolved spectral analysis on a finer temporal grid; (iv) spectral analysis of BAT and XRT simultaneous data. Hereafter, errors are reported for a $90 \%$ single parameter confidence level.

\section{Observations and data reduction}

The BAT data were analyzed using the standard BAT analysis software included in the HEAsoft 6.0.4 package and described in the Swift BAT Ground Analysis Software Manual ${ }^{2}$. BAT data from 306 to $525 \mathrm{~s}$ after the burst onset were telemetered in the masktag-lc observing mode that accumulates data in only four energy bands. As a consequence, no spectral energy analysis was performed on BAT data for this time interval.

GRB 050904 was observed by the XRT from $161 \mathrm{~s}$ up to 10 days after the burst onset, overlapping the BAT observations for about $364 \mathrm{~s}$. The XRT observation started before the end of the high energy prompt emission. Data were accumulated in WT mode up to $573 \mathrm{~s}$ after the trigger time, while all the other data were obtained in PC mode. In the WT mode only the central $8^{\prime}$ of the field of view is read out, providing one dimensional imaging and full spectral capability with a time resolution of $1.8 \mathrm{~ms}$. The PC mode provides, instead, full spatial and spectral resolution with a time resolution of $2.5 \mathrm{~s}$.

XRT data were calibrated, filtered and screened using the XRTDAS package included in the HEAsoft 6.0.4, as described in the XRT Software User's Guide ${ }^{2}$. Only observing time intervals with a CCD temperature below -47 degrees Celsius were used. The total exposures after all the cleaning procedures were $2.8 \mathrm{ks}$ and $127.3 \mathrm{ks}$ for data accumulated in the WT and PC modes, respectively. We used a 0-2 and 0-12 grade selection for data in the WT and PC modes, respectively. Such a selection provides the best combination of spectral resolution and detection efficiency. The GRB was imaged far from the CCD hot columns and no corrections for hot columns inside the photon extraction regions was necessary.

The position of the burst was refined by on-ground analysis (Palmer et al. 2005). The BAT burst position is $\mathrm{RA}_{\mathrm{J} 2000}=$ $00^{\mathrm{h}} 54^{\mathrm{m}} 53^{\mathrm{s}}, \operatorname{Dec}_{\mathrm{J} 2000}=+14^{\circ} 04^{\prime} 52^{\prime \prime}$, with an uncertainty of 2.6 . This is 3.9 from the on-board position and 0.54 from the nearIR afterglow position (Nysewander et al. 2005). The XRT afterglow position derived with xrtcentroid (v0.2.7) and including the latest boresight correction (Moretti et al. 2006) is $\mathrm{RA}_{\mathrm{J} 2000}=$ $00^{\mathrm{h}} 54^{\mathrm{m}} 50 \mathrm{~s} .8, \operatorname{Dec}_{\mathrm{J} 2000}=+14^{\circ} 05^{\prime} 09^{\prime} .0$, with an uncertainty of 3".5. The XRT derived coordinates are 35".9 from the BAT ones and 0.' 4 from the near-IR counterpart (Nysewander et al. 2005).

The BAT and XRT times are referred to the GRB 050904 onset $T=2005$ Sep. 4, 01:51:44.3 UT.

For the measured redshift $z=6.29$, the $15-350 \mathrm{keV}$ BAT band corresponds to a $109-2551 \mathrm{keV}$ band in the burst rest frame while the $0.2-10 \mathrm{keV}$ XRT band corresponds to a 1.4-73 keV band. The observed timescales are stretched by a factor $(1+z)$ with respect to the rest frame ones. In the following the GRB phenomenology is presented and discussed from the point of view of the source rest frame.

\footnotetext{
${ }^{2}$ http://swift.gsfc.nasa.gov/docs/swift/analysis/
} 


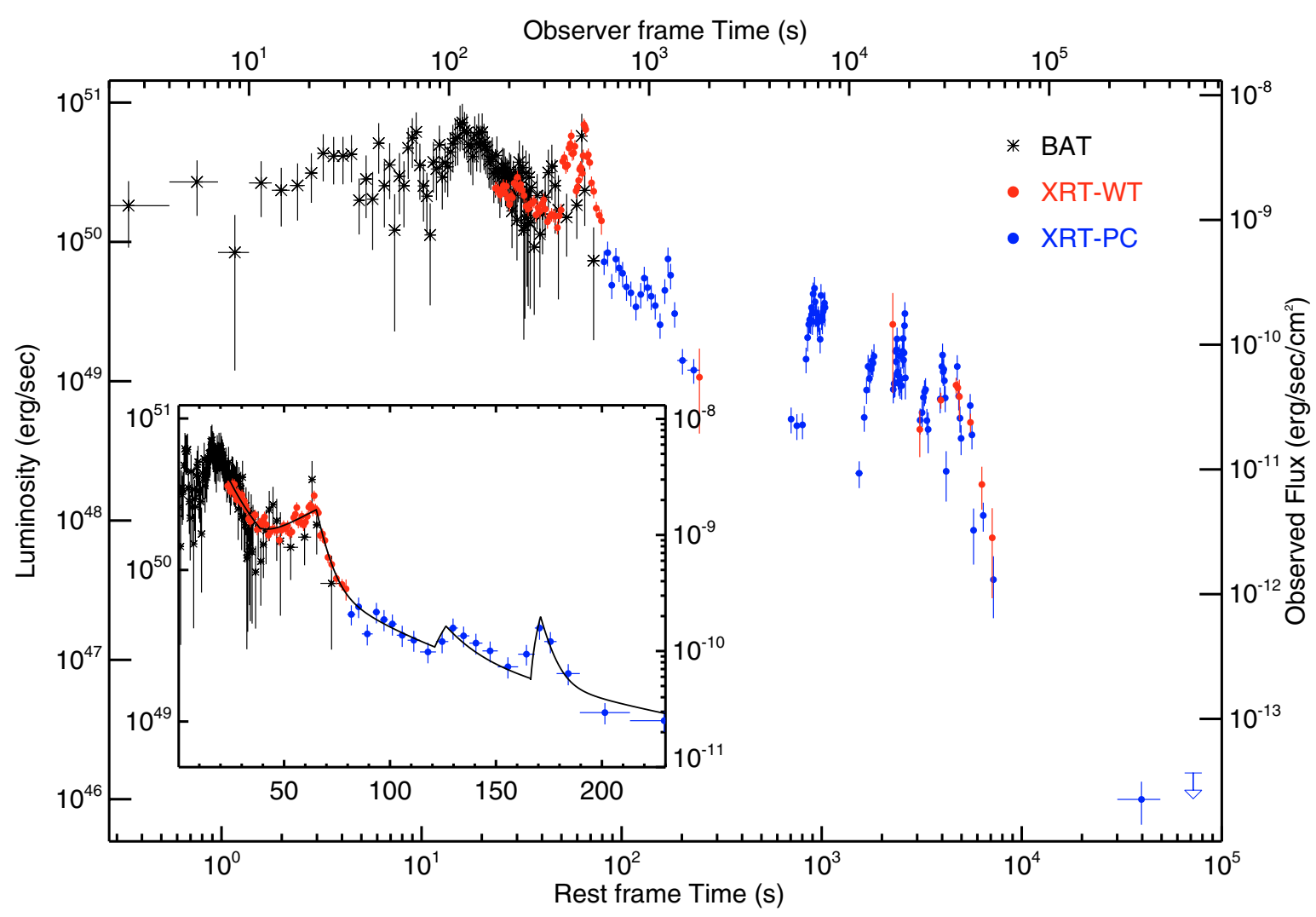

Fig. 1. The 0.2-10 keV light curve of GRB 050904 as observed by the BAT and XRT. The observed BAT count rates were extrapolated into the XRT $0.2-10 \mathrm{keV}$ band using a conversion factor evaluated from the BAT best fit spectral model (Table 1). The observed XRT count rates were converted into flux $F_{0.2-10 \mathrm{keV}}$ (scale on the right side) using the best fit spectral parameters listed in Table 1. The equivalent isotropic luminosity (scale on the left side) was calculated as $F_{0.2-10 \mathrm{keV}} 4 \pi d_{L}^{2}(1+z)^{(\Gamma-2)}$ where $d_{L}$ is the luminosity distance and the last term is the $\mathrm{K}$ correction factor for emission modeled by a power law. The error bars are given by the quadrature sum of the count rate statistical error and the estimated uncertainties in the conversion factors. The inset shows BAT and XRT $F_{0.2-10 \mathrm{keV}}$ for the first orbit and the best fit model for XRT data. Note that small differences in the the burst history shown in the inset $\left(F_{0.2-10 \mathrm{kev}}\right.$ units) and the main picture, which shows the evolution in luminosity units, are due to the photon index dependence in the $\mathrm{K}$ correction factor and its measurement in discrete time intervals.

\section{Timing analysis}

The timing analysis for the XRT data was performed by selecting events from a region centered on the source with a radius of 20 (47.2) and 35 (82.6) pixels (arcsec) for WT and PC data, respectively. The background was estimated from regions sufficiently offset ( $>2$ arcmin) from the source position to avoid contamination from the PSF wings and free from contamination by other sources.

The intensity of the source caused pile-up in the PC data up to $8 \mathrm{ks}$ from the burst onset. The pile-up correction was performed by excluding a central region of 4 pixels radius and dividing the extracted count rate by the fraction of lost point spread function (PSF, 58\%). For the rest of the observation we used the full circular extraction region.

WT data were also corrected for the fraction of PSF not included in the extraction region $(7 \%)$.

Figure 1 shows the evolution of the GRB flux and luminosity. The BAT light curve displays three main peaks: two short peaks ( $2 \mathrm{~s}$ long) at $T+3.8$ and at $T+9.3 \mathrm{~s}$, and a main longlasting peak at $\sim T+13.7 \mathrm{~s}$, where $T$ is the time of the burst onset. Emission in the BAT energy range continues up to almost $T+77.7 \mathrm{~s}$ with a weak peak at $\sim T+65 \mathrm{~s}$, coincident with the first peak of the XRT light curve. The BAT and XRT light curves overlap between $T+23$ and $T+69 \mathrm{~s}$. The early XRT light curve shows a steep decay with a slope $\alpha=-2.07 \pm 0.03$ with three flares superimposed at $T+65 \mathrm{~s}, T+126 \mathrm{~s}$ and $T+171 \mathrm{~s}$. These flares can be modeled by a linear rise lasting 26.6, 5.3 and $4.7 \mathrm{~s}$, plus an exponential decay with decay time of 4.5 , 10.98 and $5.2 \mathrm{~s}$, respectively. The best fit model of the first orbit of the XRT light curve is shown in the inset of Fig. 1. Although interrupted by observing constraints imposed by the Swift orbit, the light curve from GRB 050904 reveals highly irregular rate variations likely due to the presence of flares up to $T+1.5 \mathrm{~h}$. At later times the flaring activity is not detected and only a residual emission, $10^{5}$ times lower than the initial intensity, is visible. Note that the XRT light curve presented in Watson et al. (2005) shows an evident discontinuity at the end of the WT observation segment likely caused by an error in his flux conversions for the following PC mode data, all of which are systematically too low by about a factor of four.

Figure 2 shows the rest frame XRT light curves of GRB 050904 in the 1.4-14 keV and 14-73 keV bands (top and middle panels). The two light curves are binned before conversion to the rest frame in order to have at least 40 counts per bin in both bands. The hardness ratio (bottom panel) is defined as $\mathrm{H} / \mathrm{S}$, where $\mathrm{H}$ (hard) and $\mathrm{S}$ (soft) are the high energy and the low energy bands. The $\mathrm{H} / \mathrm{S}$ plot reveals a significant shift to softer energies with time during the first $80 \mathrm{~s}$, with the exception of the flaring episode at $T+65 \mathrm{~s}$, where the hardness ratio peaks, too. Later emission shows no evidence of further softening until the tail of the last flare around $T+5500 \mathrm{~s}$. 
Table 1. BAT and XRT spectral analysis results. The BAT fluxes in the XRT band are extrapolated from the best fit models. Quoted errors are at the $90 \%$ confidence level.

\begin{tabular}{|c|c|c|c|c|c|c|c|}
\hline \multirow[t]{2}{*}{ Interval } & \multicolumn{2}{|c|}{ Time (s) } & \multirow{2}{*}{$N_{\mathrm{H}}\left(10^{22} \mathrm{~cm}^{-2}\right)$} & \multirow[t]{2}{*}{$\Gamma$} & \multirow{2}{*}{$\begin{array}{l}\chi_{v}^{2} \text { (d.o.f.) } \\
0.2-10 \mathrm{keV}\end{array}$} & \multirow{2}{*}{\multicolumn{2}{|c|}{$\begin{array}{l}\text { Flux }\left(10^{-9} \mathrm{erg} \mathrm{cm}^{-2} \mathrm{~s}^{-1}\right) \\
15-350 \mathrm{keV}\end{array}$}} \\
\hline & Start & Stop & & & & & \\
\hline BAT 1 & -1.43 & 2.69 & $\overline{-}$ & $-1.2 \pm 0.4$ & $1.2(57)$ & 1.4 & 22.9 \\
\hline 2 & 2.69 & 4.89 & _- & $-1.05 \pm 0.16$ & $0.86(57)$ & 3.2 & 90.8 \\
\hline 3 & 4.89 & 10.1 & - & $-1.36 \pm 0.21$ & 0.97 (57) & 3.4 & 30.9 \\
\hline 4 & 10.1 & 20.4 & - & $-1.17 \pm 0.08$ & $0.95(57)$ & 3.6 & 66.8 \\
\hline 5 & 20.4 & 30.6 & - & $-1.22 \pm 0.10$ & $0.93(57)$ & 3.0 & 45.7 \\
\hline 6 & 30.6 & 41.6 & - & $-1.5 \pm 0.3$ & $0.88(57)$ & 2.0 & 9.9 \\
\hline XRT 1 & 23.2 & 28.7 & $5.73 \pm 4.2$ & $-1.19 \pm 0.1$ & $0.77(62)$ & 3.5 & - \\
\hline 2 & 28.7 & 36.9 & $5.5 \pm 2.5$ & $-1.34 \pm 0.08$ & $0.98(95)$ & 2.5 & - \\
\hline 3 & 36.9 & 50.6 & $3.4 \pm 2.2$ & $-1.33 \pm 0.08$ & $0.78(89)$ & 1.3 & - \\
\hline 4 & 50.6 & 58.8 & $7.7 \pm 4.5$ & $-1.85 \pm 0.1$ & $1.12(56)$ & 1.4 & - \\
\hline 5 & 58.8 & 67.1 & $4.2 \pm 2.0$ & $-1.50 \pm 0.09$ & $1.14(73)$ & 1.7 & - \\
\hline 6 & 67.1 & 79.8 & $1.5 \pm 1.4$ & $-1.86 \pm 0.13$ & $0.94(37)$ & 0.54 & - \\
\hline 7 & 79.8 & 159.4 & $<6.4$ & $-1.80 \pm 0.15$ & $1.12(23)$ & 0.12 & - \\
\hline 8 & 159.4 & 244.4 & $<6.4$ & $-1.97 \pm 0.24$ & $0.90(7)$ & 0.05 & - \\
\hline 9 & 628 & 848 & $<5.2$ & $-1.80 \pm 0.24$ & $0.92(7)$ & 0.02 & - \\
\hline 10 & 848 & 1040 & $<6.8$ & $-1.86 \pm 0.14$ & $0.90(35)$ & 0.08 & - \\
\hline 11 & 1452 & 1863 & $<5.8$ & $-2.01 \pm 0.22$ & 0.80 (17) & 0.02 & - \\
\hline 12 & 2275 & 2618 & $<6.9$ & $-1.90 \pm 0.14$ & $1.26(47)$ & 0.04 & - \\
\hline 13 & 3045 & 8173 & $<4.0$ & $-1.97 \pm 0.12$ & $1.24(35)$ & 0.008 & - \\
\hline
\end{tabular}

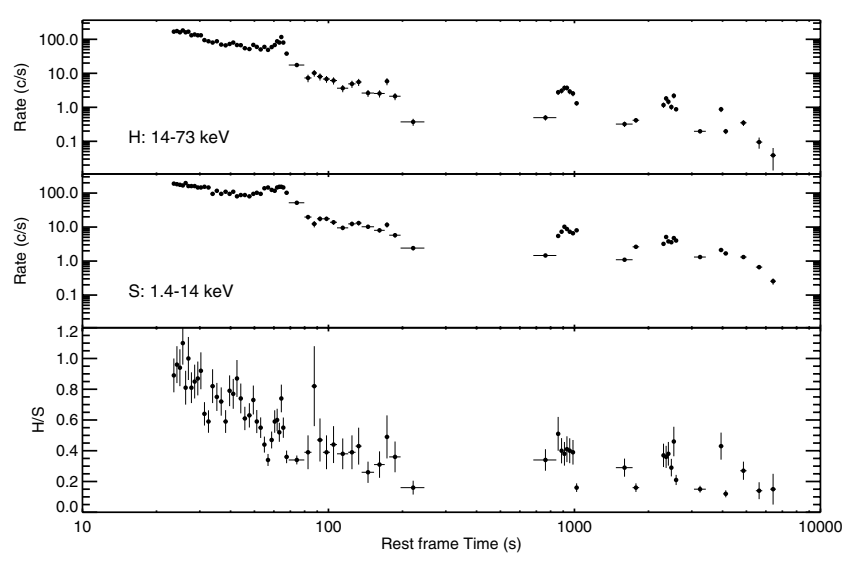

Fig. 2. Hardness ratio evolution of GRB 050904. The top and middle panels show the count rate evolution of XRT data in two different energy bands in the rest frame.

\section{Spectral analysis}

The spectral analysis of GRB 050904 was performed by selecting two sets of time intervals for the BAT and XRT observations, corresponding to characteristic phases of the light curve evolution. The BAT spectra were accumulated in the $14-150 \mathrm{keV}$ observed band in six time intervals up to $41.6 \mathrm{~s}$ from the burst onset (Table 1). No BAT spectrum was accumulated from $T+41.6$ to $T+72 \mathrm{~s}$ because BAT data were in the masktag-lc observing mode with only four energy bands. The XRT spectra were accumulated in thirteen time intervals from $T+23 \mathrm{~s}$ to $T+8173 \mathrm{~s}$ from the burst onset (Table 1). The WT and PC spectra were extracted from the same regions used for the timing analysis. Instrumental energy channels below $0.3 \mathrm{keV}$ and above $10 \mathrm{keV}$ for PC and WT spectra were ignored and the background was evaluated in regions sufficiently offset ( $>2$ arcmin) from the source position to avoid contamination from the PSF wings and free of contamination from other sources in the field of view. Moreover, energy channels between 0.5 and $0.6 \mathrm{keV}$ were excluded because of the presence of a sistematics in such a range due to a time dependent energy scale problem at low energy ${ }^{3}$; a sistematics in the gain offset produces a negative residual in the $0.5 \mathrm{keV}$ region increasing in the fit procedure the $N_{\mathrm{H}}$ estimation. The BAT spectra were modeled with a power law with photon index $\Gamma\left(F(E) \propto E^{\Gamma+1}\right)$ while the XRT spectra were modeled with a power law plus two absorption components: one for the intrinsic absorption in the host galaxy and one for the Galactic absorption. The latter was fixed to the line-of-sight value of $4.93 \times 10^{20} \mathrm{~cm}^{-2}$ (Dickey \& Lockman 1990). The model gave acceptable $\chi_{v}^{2}$ values for all the selected intervals (Table 1). More complex models, such as a Band function (Band et al. 1993), cannot be constrained by the data. Figure 3 shows the evolution with time of the photon index $\Gamma$. The BAT spectra have $\Gamma \sim-1.2$, consistent with typical values of the $\alpha_{\text {Band }}$ parameter of the Band model (Preece et al. 2000). This strongly suggests that the BAT observes the low energy part of the Band function and that the peak energy of the GRB spectrum is above $150 \times(1+z) \mathrm{keV}$ in the source rest frame. If we exclude the spectrum of the first XRT flare at $T+65 \mathrm{~s}$, the XRT photon indices show a clear decreasing trend from about -1.2 to about -1.9 in the first $T+200 \mathrm{~s}$. No further spectral evolution is present in later XRT data, in agreement with the hardness ratio curve. The BAT and XRT photon indices are in good agreement in the overlapping region. We also detected in the XRT WT spectra $(T<T+67.1 \mathrm{~s})$, a highly significant absorption in excess of the Galactic value. The intrinsic absorption column decreases with time at high significant level. In the time intervals where the burst was observed in PC mode $(T>T+67.1 \mathrm{~s})$, due to the decreased statistics, only upper limits were measured. Table 1 shows the best fit parameters for each of the selected time intervals.

The GRB 050904 spectral evolution is also quite evident in Fig. 4 where the $E^{2} \mathrm{~F}(\mathrm{E})$ deconvolved spectra (equivalent to $v \mathrm{~F}(v)$ spectra) selected in 5 ad hoc intervals (bottom panel in Fig. 3) are shown together with the best fit spectral models. The first spectrum (A) is accumulated in the time interval $T-1.43 \mathrm{~s}-T+23.2 \mathrm{~s}$, when only BAT observed the burst. The second spectrum (B) is from $T+23.2 \mathrm{~s}$ to $T+41.7 \mathrm{~s}$ when the GRB is simultaneously observed by BAT and the

\footnotetext{
${ }^{3}$ http://heasarc.gsfc.nasa.gov/docs/swift/analysis/
} 
Table 2. BAT and XRT spectral analysis results of relative to 5 ad hoc selected intervals of the GRB evolution (see bottom panel in Fig. 3). The BAT fluxes in the XRT band are extrapolated from the best fit models. Quoted errors and upper limits are at the $90 \%$ confidence level.

\begin{tabular}{crrrccccc}
\hline \hline & Interval & \multicolumn{2}{c}{ Time (s) } & \multirow{2}{*}{$N_{\mathrm{H}}\left(10^{22} \mathrm{~cm}^{-2}\right)$} & $\Gamma$ & \multirow{2}{*}{$\chi_{v}^{2}$ (d.o.f.) } & \multicolumn{2}{c}{ Flux $\left(10^{-9} \mathrm{erg} \mathrm{cm}^{-2} \mathrm{~s}^{-1}\right)$} \\
& & Start & Stop & & & & & \\
$0.2-10 \mathrm{keV}$ & $15-350 \mathrm{keV}$ \\
\hline BAT & $\mathrm{A}$ & -1.43 & 23.2 & - & $-1.19 \pm 0.07$ & $0.96(57)$ & 3.1 & 52.8 \\
BAT+XRT & $\mathrm{B}$ & 23.2 & 41.6 & $7.5 \pm 1.5$ & $-1.37 \pm 0.02$ & $1.0(189)$ & 2.6 & 11.9 \\
XRT & $\mathrm{C}$ & 41.6 & 82.3 & $4.7 \pm 1.2$ & $-1.63 \pm 0.05$ & $1.19(205)$ & 1.33 & - \\
XRT & $\mathrm{D}$ & 82.3 & 224 & $<2.1$ & $-1.7 \pm 0.1$ & $1.24(22)$ & 0.03 & - \\
XRT & $\mathrm{E}$ & 628 & 8163 & $<2.9$ & $-1.74 \pm 0.07$ & $1.243(80)$ & 0.006 & - \\
\hline
\end{tabular}

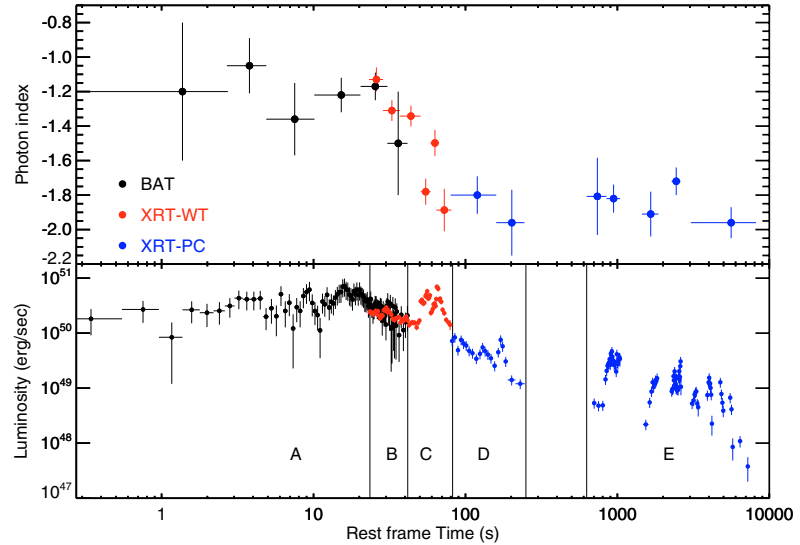

Fig. 3. Spectral evolution of GRB 050904. The top panel illustrates how the photon index $\Gamma$ of GRB 050904 changes during the observation. In the bottom panel the burst evolution is plotted to show how the time intervals for spectral analysis were selected. Vertical bars indicate the time intervals selected for BAT and XRT spectral analysis whose results are reported in Table 2 and Fig. 4.

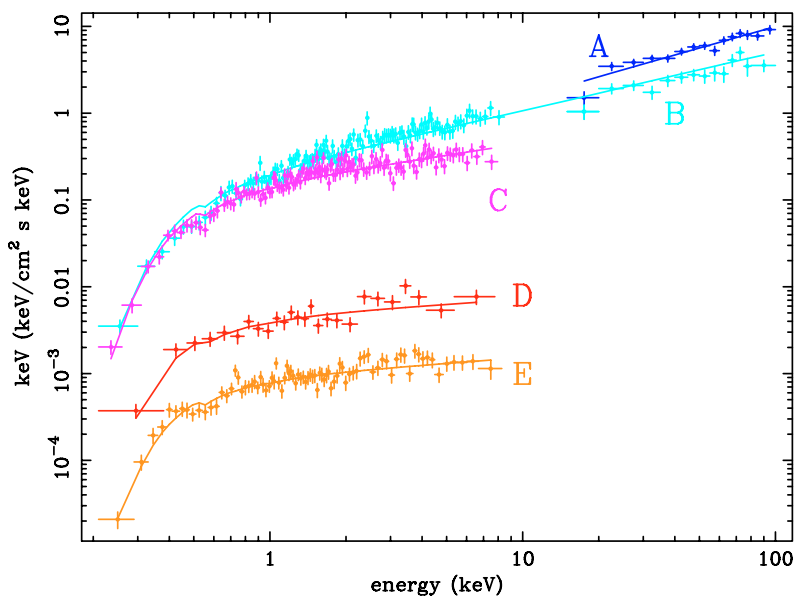

Fig. 4. Spectral evolution of GRB 050904; BAT and XRT $E^{2} \mathrm{~F}(\mathrm{E})$ spectra (equivalent to $v \mathrm{~F}(v)$ spectra) deconvolved from the detector response for five selected time intervals (see bottom panel in Fig. 3).

XRT. The following spectra $(\mathrm{C}, \mathrm{D}, \mathrm{E})$ refer to the time intervals $T+41.6 \mathrm{~s}-T+82.3 \mathrm{~s}, T+82.3 \mathrm{~s}-T+224 \mathrm{~s}$ and $T+628 \mathrm{~s}-T+8163$, respectively, when only the XRT detected the burst. The energy distribution is clearly softening with time starting from interval $\mathrm{C}$. The fit in the broad energy band of interval $\mathrm{B}$ is also well modeled by an absorbed power law while a Band function (Band et al. 1993), cannot be constrained by the data. Table 2 shows the best fit parameters for each of the selected time intervals.

We also evaluated the contribution to the total fluence in the $1.4-73 \mathrm{keV}$ band of the three flares $(T+65, T+126$ and $T+171 \mathrm{~s})$ superimposed on the early XRT light curve. The fluence values over the continuum are $(1.2 \pm 0.08) \times 10^{-6},(4.7 \pm 0.5) \times 10^{-8}$ and $(5.8 \pm 0.6) \times 10^{-8} \mathrm{erg} \mathrm{cm}^{-2}$, respectively. The fluence of the XRT continuum over the first orbit (i.e. from 23.2 to $244.4 \mathrm{~s}$ ) is $(4.9 \pm 0.3) \times 10^{-6} \mathrm{erg} \mathrm{cm}^{-2}$. The extrapolated $1.4-73 \mathrm{keV}$ BAT fluence in the time interval from the burst onset to the start of the XRT observation is $(4.1 \pm 0.2) \times 10^{-6} \mathrm{erg} \mathrm{cm}^{-2}$. The three XRT flares are therefore $5 \%, 1 \%$ and $1 \%$ of the total $1.4-73 \mathrm{keV}$ emission observed up to $T+244 \mathrm{~s}$. The $1.4-73 \mathrm{keV}$ fluence in the remaining part of the XRT observation is $1.8 \times 10^{-6} \mathrm{erg} \mathrm{cm}^{-2}$. This value is only a lower limit because of the observing gaps.

\section{Discussion}

GRB 050904 was a long, multi-peaked, bright GRB with strong $\mathrm{X}$-ray flaring activity lasting up to $1-2 \mathrm{~h}$ in the source rest frame (or $5-6 \times 10^{4} \mathrm{~s}$ in the observer frame). X-ray flares are detected in nearly half of the Swift long bursts (e.g. GRB 050406 (Burrows et al. 2005a; Romano et al. 2006), GRB 050502 (Burrows et al. 2005a; Falcone et al. 2006), GRB 050607 (Pagani et al. 2006), GRB 050713A (Morris et al. 2006)). The rise time and decay time of these flares seen at lower $z$ are frequently very fast with a ratio between the duration and peak time $\delta t / t \ll 1$. These features make difficult to explain these flares with mechanisms associated with the external shock (Zhang et al. 2006)

The variability of GRB 050904 X-ray light curve is even more dramatic than the typical Swift afterglow showing flares, and the amplitude and rise/fall times of the flares are consistent with the behavior of nearby $(z \leq 1)$ long GRBs (Fishman $\&$ Meegan 1995). This suggests to interpret them as late internal shocks related to central engine activity. In this scenario they would have the same origin as the prompt gamma-ray emission (Zhang et al. 2006; Nousek et al. 2006; O'Brien et al. 2006). This would require that the central engine remain active up to at least $5000 \mathrm{~s}$, consistent with the collapsar model (MacFadyen et al. 2001), which allows central engine activity for up to a few hours. Then, BAT and XRT have likely recorded a single continuous observation of long lasting prompt emission where the standard X-ray afterglow component is hardly detectable because its contribution is drowned by the intense and long lasting activity of the central engine.

TAROT observed a flare in the optical band (Boër et al. 2006; Gendre et al. 2006) which is simultaneous with the X-ray flare at $T+65 \mathrm{~s}$. Later $(>T+1200 \mathrm{~s})$ optical and infrared observations (Tagliaferri et al. 2005b) are too sparse to test if the flaring activity observed at later times in X-rays is indeed present. The multiwavelength fit by Tagliaferri et al. (2005b) suggests that the optical data taken after $T+1200 \mathrm{~s}$ are in agreement with standard afterglow emission and jet lateral spreading at about $T+80 \mathrm{ks}$. Physical interpretations to explain the simultaneous X-ray and optical flare at $T+65 \mathrm{~s}$ are extensively discussed in Gendre et al. (2006). They showed that delayed external shock from a 
thick shell (Piro et al. 2005), synchrotron emission from reverse shock (Fan \& Wei 2005), inverse Compton emission from reverse shock (Kobayashi et al. 2005) and inverse Compton from late internal shock (Wei et al. 2006) cannot satisfactory explain the simultaneous X-ray and optical flare. On the other hand, the late internal shock model proposed by Zou et al. (2006) could account for the broad band data of the first flare.

In the time interval from $T+23$ to $T+244 \mathrm{~s}$, the observed intensity underlying the XRT flares decays as $t^{\alpha}$ with $\alpha=-2.07 \pm 0.03$. An initial steep decay of the X-ray emission has been observed in many other GRBs detected by Swift (Tagliaferri et al. 2005a; Nousek et al. 2006; O'Brien et al. 2006). The decay slope together with the XRT energy index $\beta=\Gamma+1 \sim-0.2$ measured up $T+50 \mathrm{~s}$ are in good agreement with the interpretation of the observed emission as due to highlatitude emission (Kumar \& Panaitescu 2000). In this model, the tail of a peak is the emission from the shocked gas moving at angle $\theta>1 / \gamma$ (where $\gamma$ is the Lorentz factor) relative to the observer. The higher the angle $\theta$, the later the photon arrival-time and the weaker the relativistic beaming of the emission, leading to a $t^{\alpha}$ decay with $\alpha=\beta-2$. After $T+50 \mathrm{~s}$, due to the decrease of $\beta$ to about -0.7 , the predicted slope would be steeper than the measured -2 . This deviation could be reconciled with the high-latitude emission assuming that the delayed radiation from the outer parts of the emitting curved shell is softer and brighter than the radiation along our line of sight (Kumar et al. 2006).

The decrease of the photon index around $T+50 \mathrm{~s}$ could be interpreted as an indication of a shift of the spectral peak en$\operatorname{ergy}\left(E_{\mathrm{p}}\right)$ towards lower energies, but poor statistics and the narrowness of the XRT energy range do not allow us to verify this hypothesis.

We detected highly significant absorption in excess of the Galactic value. The host column density decreases with time at a high significance level. This is consistent with the idea that the circum-burst absorbing material is photoionized by the highenergy photons of the burst (Perna \& Loeb 1998). Evidence for such a decrease has been found for GRB 980329 (Frontera et al. 2000) and GRB000528 (Frontera et al. 2000) but neither of them has a comparable data quality. We do not confirm evidence found by Watson et al. (2006) of an increase of the column density at the peak of the first X-ray flare.

Our lack of knowledge concerning the peak energy of the BAT and XRT spectra does not allow a precise estimate of the total energy released by GRB 050904 . However, we can calculate lower and upper limits to the isotropic-equivalent radiated energy $E_{\text {iso }}$ up to $244 \mathrm{~s}$ from the burst onset, i.e. including contributions from the first three XRT flares. To evaluate the lower limit to $E_{\text {iso }}$ we integrated the best fit power law spectral energy distributions in the $(1-200) \times(1+z) \mathrm{keV}$ band and in the $(1-10) \times(1+z) \mathrm{keV}$ band for the BAT and XRT, respectively. The standard energy range $1-10^{4} \mathrm{keV}$ (rest frame) was used to evaluate the upper limit to $E_{\text {iso }}$. We obtained $6.6 \times 10^{53} \mathrm{erg}<E_{\text {iso }}<$ $3.2 \times 10^{54} \mathrm{erg}$. Additional contributions from the later flare portions are only a few percent in both the upper and lower limit. The large isotropic equivalent energy of this burst is in agreement with the Amati relation (Amati et al. 2002) with an $E_{\mathrm{p}}$ of about $1500 \mathrm{keV}$ in the rest frame. This is consistent with our non-detection of a peak energy in BAT spectral fit.

The break observed in the optical and infrared afterglows (Tagliaferri et al. 2005b) at $T_{\mathrm{b}}=2.6 \pm 1.0$ days (observer frame) and the range of $E_{\text {iso }}$ evaluated above imply a jet halfopening angle $\vartheta_{\text {jet }}$ between $2^{\circ}$ and $4^{\circ}$, assuming a radiative efficiency $\eta=20 \%$ and a circumburst medium density $n=$ $3 \mathrm{~cm}^{-3}$. This angle estimate is consistent with those obtained by

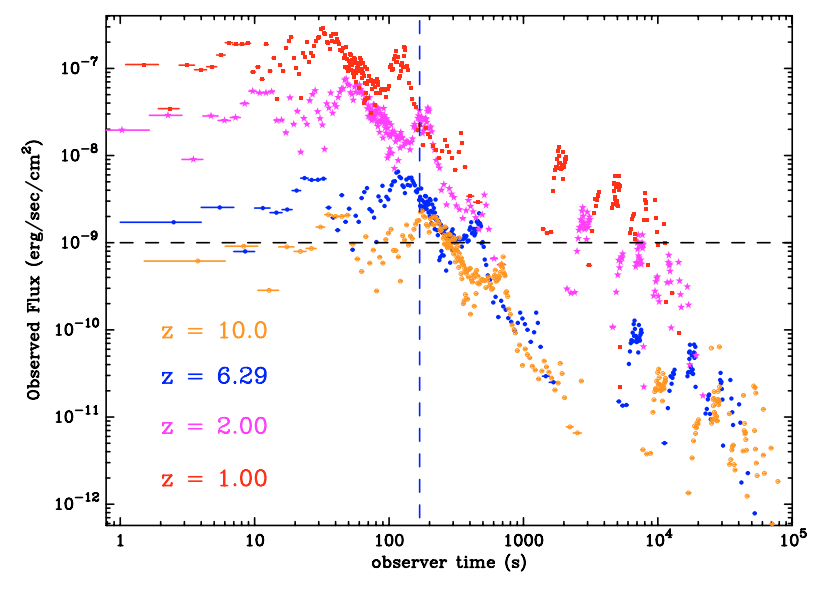

Fig. 5. GRB 050904 light curves in the $0.2-10 \mathrm{keV}$ band as they would be observed if the burst were located at different redshifts. Each light curve includes both BAT and XRT extrapolations. The horizontal line gives an indication of the sensitivity limit of BAT, while the vertical line marks the start of the XRT follow-up in the observing frame.

modelling optical light-curve breaks observed in 10 pre-Swift GRBs (Panaitescu 2005). It corresponds to a collimationcorrected energy $E_{\gamma}$ between 0.4 and $4.4 \times 10^{51} \mathrm{erg}$. This is well within the $E_{\gamma}$ distribution of GRBs with known redshift (Frail et al. 2001; Bloom et al. 2003). Consistency with the Ghirlanda relation (Ghirlanda et al. 2004) constrains the rest frame peak energy of the average spectrum to be between 560 and $1300 \mathrm{keV}$.

In the optical afterglow, the measured pre-break slope decay $\alpha_{\mathrm{o}}=-0.7 \pm 0.2$ (Tagliaferri et al. 2005) requires an electron index of $p=(4 / 3) \alpha_{\mathrm{o}}+1 \sim 1.9 \pm 0.3$ in a local uniform interstellar medium. The expected intrinsic optical spectral slope should be $\beta_{\mathrm{o}}=(p-1) / 2 \sim-0.45 \pm 0.15$ while the observed one is $\beta_{0}=-1.25 \pm 0.15$ (Tagliaferri et al. 2005). This discrepancy could be naturally explained assuming the presence of a bit of dust $\left(A_{V}=(1.25-0.45) /(1+z)=0.1\right)$ in the local host medium. In the X-ray regime the slowest decay of a possible underlying continuum, inferred by fitting the lowest points in Fig. 1 after $T+1000 \mathrm{~s}$, is $\alpha_{\mathrm{x}} \sim-1.2$. This is marginally consistent with the slope expected for the forward shock emission for the value of electron index inferred from optical data: $\alpha_{\mathrm{x}}=(3 p-2) / 4 \sim 0.95 \pm 0.20$, under the hypothesis that the cooling frequency is between optical and X-rays. The expected spectral index in $\mathrm{X}$-rays is $\beta_{\mathrm{x}}=p / 2 \sim-1.0 \pm 0.1$, consistent with the observed $\beta_{\mathrm{x}}=-1.0 \pm 0.2$ after $T+1000 \mathrm{~s}$. The continuum component inferred by the fitting the the lowest points in Fig. 1 is, therefore, marginally consistent with a standard forward shock emission and the properties of the optical emission reported by Tagliaferri et al. (2005). However, we cannot prove that the estimated underlying X-ray continuum and the late optical light curve are the same forward shock emission because the observational gaps do not allow to see the minima of the X-ray light curve.

Figure 5 shows how GRB 050904 would appear in the Swift $0.2-10 \mathrm{keV}$ band if it were at redshifts other than $z=6.29$. The observed intensity varies inversely with the square of the $(1+z)$ factor. A further dependence on $z$ is due to the K correction that accounts for the redshift dependence of the luminosity in a given wavelength band. The observed timescale undergoes different stretching factors with respect to the rest frame: the burst would appear longer at higher redshift. The horizontal line in Fig. 5 gives an indication of the sensitivity limit of BAT, while the vertical line marks the start of the XRT follow-up. Starting 


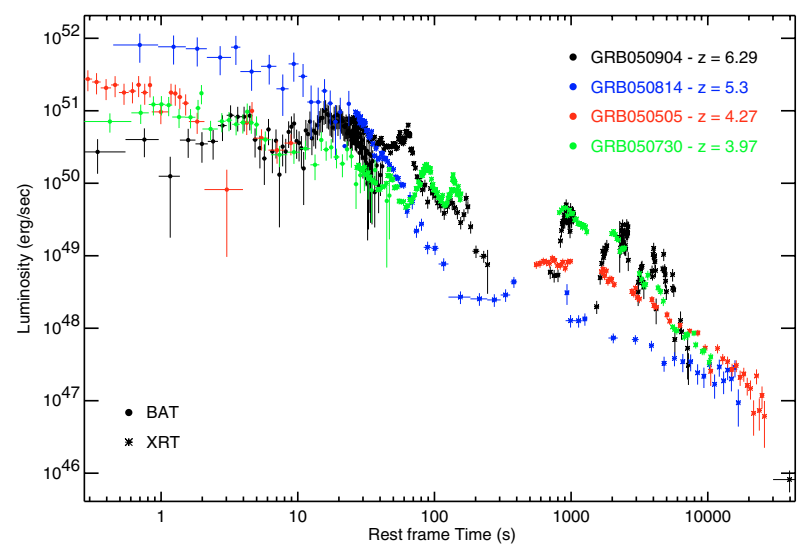

Fig. 6. The K-corrected $0.2-10 \mathrm{keV}$ time histories of the high-redshift (‡4) GRBs observed by Swift. Each light curve includes both XRT and BAT data extrapolated to the XRT energy range. The K correction was performed for an average photon index for simplicity.

from a redshift lower than $\sim 2$, BAT would have observed all later flares. GRB 050904 would have been detected up to a distance corresponding to $z \sim 10$. Comparing the intensity of other GRBs observed by Swift (see Fig. 3 in O'Brien et al. 2006) with the flux that we would observe if GRB 050904 had exploded at a redshift lower than 2 ( $\sim$ the average redshift of the Swift GRBs) we see that this burst was intrinsically bright in X-rays.

After one year of operations, Swift has detected four confirmed high-redshift $(\gtrsim 4)$ GRBs (out of 24 with known redshifts). Figure 6 shows the K-corrected $0.2-10 \mathrm{keV}$ luminosity evolution of these GRBs, including both BAT and XRT data set. BAT light curves are obtained by extrapolation of the $15-150 \mathrm{keV}$ light curve to the XRT energy band. In addition to GRB 050904 this sample includes GRB 050730 at $z=3.969$ (Chen et al. 2005), GRB 050505 at $z=4.27$ (Berger et al. 2006) and GRB 050814 at $z=5.3$ (Jakobsson et al. 2006). All of the bursts are exceptionally luminous and long-lasting (as measured in the source rest-frame) and are among the brightest GRBs ever observed. Their exceptional intensity is not due to selection effects, since the 15-150 keV burst fluxes are well above the BAT detection threshold. Rather, their unique properties could be due to their likely origin within some of the first star-forming regions in the Universe; Woosley \& Heger (2006) suggested that the very low metallicities of the progenitors at these epochs may provide an explanation. A more reliable conclusion about systematic differences and similarities in luminosities and durations of high redshift GRBs will require an increase in the sample size, which should come in future years of Swift operation.

Detecting high-redshift GRBs with Swift, and measuring their redshifts with ground-based spectroscopy, is of substantial interest because of the link between long-duration GRBs and the star formation rate. The GRBs with measured redshift can be used to infer the cosmological star formation history, with relatively minor (or in any case unique) selection effects by comparison to other methods (Porciani \& Madau 2001; Lamb \& Reichart 2000; Bromm \& Loeb 2002; Natarajan et al. 2005). A preliminary estimate of the star formation rate derived from Swift bursts (Price et al. 2006) shows, within current broad uncertainties, a flat or (at the highest redshifts) slowly-declining star formation rate, consistent with results obtained from color-selected galaxy observations (Bunker et al. 2004).

\section{Summary and conclusion}

We have presented the results of the analysis of BAT and XRT observations of the high redshift $(z=6.29)$ GRB 050904. The GRB light curve is characterized by the presence of a long flaring activity lasting up to 1-2 h after the burst onset in the burst rest frame, with no evidence of a smooth power-law decay following the prompt emission as seen in other GRBs. We interpret the overall phenomenology of GRB 050904 as long lasting prompt emission where the standard X-ray afterglow component is hardly detectable because its contribution is drowned by the intense and long lasting activity of the central engine. The spectral energy distribution softens with time, with the photon index decreasing from -1.2 during the BAT observation to -1.9 at the end of the XRT observation. The dips of the late X-ray flares may be consistent with an underlying X-ray emission arising from the forward shock and with the properties of the optical afterglow reported by Tagliaferri et al. (2005b).

Highly significant absorption in excess of the Galactic value has been detected. The intrinsic hydrogen-equivalent colunm density shows a significant decreasing with time that we interpret as due to the photoionization of the circum-burst absorbing material by the high-energy photons of the burst causing a graduale reduction of the opacity.

We have calculated lower and upper limits to the isotropicequivalent radiated energy $E_{\text {iso }}$ up to 244 s from the burst onset, i.e. including contributions from the first three XRT flares. We obtained $6.6 \times 10^{53} \mathrm{erg}<E_{\text {iso }}<3.2 \times 10^{54} \mathrm{erg}$. This range of $E_{\text {iso }}$ and the break observed in the optical and infrared afterglows imply a jet half-opening angle $\vartheta_{\text {jet }}$ between $2^{\circ}$ and $4^{\circ}$, assuming a radiative efficiency $\eta=20 \%$ and a circumburst medium density $n=3 \mathrm{~cm}^{-3}$.

Acknowledgements. The authors acknowledge support from ASI, NASA and PPARC. K.H. is grateful for support under NASA grant FDNAG5-9210. We also acknowledge the anonymous referee for very useful suggestions.

\section{References}

Amati, L., Frontera, F., Tavani, M., et al. 2002, A\&A, 390, 81 Andersen, M. I., Hjorth, J., Pedersen, H., et al. 2000, A\&A, 364, L54 Band, D., Matteson, J., Ford, L., et al. 1993, ApJ, 413, 281 Barthelmy, S., Barbier, L. M., Cummings, J. R., et al. 2005a, Space Sci. Rev., 120,143

Barthelmy, S. D., Cannizzo, J. K., Gehrels, N., et al. 2005b, ApJ, 635, 133 Barthelmy, S. D., Chincarini, G., Burrows, D. N., et al. 2005c, Nature, 438, 994 Becker, R. H., Fan, X., White, R. L., et al. 2001, AJ, 122, 2850 Berger, E., Penprase, B. E., Cenko, S. B., et al. 2006, ApJ, 642, 979 Bloom, J. S., Kulkarni, S. R., \& Djorgovski, S. G. 2002, AJ, 123, 1111 Bloom, J. S., Frail, D. A., Kulkarni, S. R., et al. 2003, ApJ, 594, 674 Bromm, V., \& Loeb, A. 2002, ApJ, 575, 111

Boër, M., Atteia, J. L., Damerdji, Y., et al. 2006, ApJ, 638, L71

Bunker, A. J., Stanway, E. R., Ellis, R. S., \& McMahon, R. G. 2004, MNRAS, 355,374

Burrows, D. N., Romano, P., Falcone, A., et al. 2005a, Science, 309, 1833

Burrows, D. N., Hill, J. E., Nousek, A., et al. 2005b, Space Sci. Rev., 120, 165

Burrows, D. N., Romano, P., Godet, O., et al. 2005c, in Proc. The X-ray Universe 2005, ESA-SP-604, 877

Campana, S., Tagliaferri, G., Lazzati, D., et al. 2006, A\&A, 454, 113 Chen, H. S., Prochaska, J. X., Bloom, J. S., \& Thompson, I. B. 2005, ApJ, 634, L25

Costa, E., Frontera, F., Heise, J., et al. 1997, Nature, 387, 783

Cucchiara, A., Cummings, J., Holland, S., et al. 2005, GCN Circ., 3923

Cummings, J., Angelini, L., Barthelmy, S. D., et al. 2005, GCN Circ., 3910

Cusumano, G., Mangano, V., Angelini, L., et al. 2006a, ApJ, 639, 316

Cusumano, G., Mangano, V., Chincarini, G., et al. 2006b, Nature, 440, 164

Dickey, J. M., \& Lockman, F. J. 1990, ARA\&A, 28, 215

Falcone, A. D., Burrows, D. N., Lazzati, D., et al. 2006, ApJ, 641, 1010

Fan, X., Strauss, M. A., Schneider, D. P., et al. 2003, AJ, 125, 1649

Fan, Y. Z., \& Wei, D. M. 2005, MNRAS, 364, L42 
Fishman, G. J., \& Meegan, C. A. 1995, ARA\&A, 33, 415

Fox, D. B., Frail, D. A., Price, P. A., et al. 2005, Nature, 437, 845

Frail, D. A., Kulkarni, S. R., Nicastro, S. R., et al. 1997, Nature, 389, 261

Frail, D. A., Kulkarni, S. R., Sari, R., et al. 2001, ApJ, 562, L55

Frontera, F., Amati, L., Costa, E., et al. 2000, ApJS, 127, 59

Frontera, F., Amati, L., Lazzati, D., et al. 2004, ApJ, 614, 301

Gehrels, N., Chincarini, G., Giommi, P., et al. 2004, ApJ, 611, 1005

Gendre, P., Galli, A., Corsi, A., et al. 2006, A\&A, submitted [arXiv:astro-ph/0603431]

Ghirlanda, G., Ghisellini, G., \& Lazzati, D. 2004, ApJ, 616, 331

Kawai, N., Yamada, T., Kosugi, G., et al. 2005, GCN Circ., 3937

Kneib, J. P., Ellis, R. S., Santos, M. R., \& Richard, J. 2004, ApJ, 607, 697

Kobayashi, S., Zhang, B., Mészáros, P., \& Burrows, D. N. 2005, ApJ, submitted [arXiv: astro-ph/0506157]

Kumar, P., \& Panaitescu, A. 2000, ApJ, 541, L92

Kumar, P., McMahon, E., Barthelmy, S. D., et al. 2006, MNRAS, 367, L52

Jakobsson, P., Leva, A., Gynbo, J. P., et al. 2006, A\&A, 447, 897

Lamb, D. Q., \& Reichart, D. E. 2000, ApJ, 536, 1

MacFadyen, A. I., Woosley, S. E., \& Heger, A. 2001, ApJ, 550, 410

Mészáros, P., \& Rees, M. J. 1997, ApJ, 476, 232

Moretti, A., Perri, M., Capalbi, M., et al. 2006, A\&A, 448, L9

Morris, D. C., Reeves, J., Pal'shin, V., et al. 2006, ApJ, submitted [arXiv: astro-ph/0602490]

Natarajan, P., Albanna, B., Hjorth, J., et al. 2005, MNRAS, 364, L8

Nousek, J. A., Kouveliotou, C., Groupe, D., et al. 2006, ApJ, 642, 389

Nysewander, M., Reichart, D., \& Haislip, J. 2005, GCN Circ., 3922
O’Brien, P. T., Willingale, R., Osborne, J., et al. 2006, A\&A, in press [arXiv: astro-ph/0601125]

Pagani, C., Morris, D. C., Kobayashi, S., et al. 2006, ApJ, 645, 1315

Palmer, D., Barbier, L., Barthelmy, S., et al. 2005, GCN Circ., 3918

Panaitescu, A. 2005, MNRAS, 363, 1409

Perna, R., \& Loeb, A. 1998, ApJ, 501, 467

Piro, L., De Pasquale, M., Soffitta, P., et al. 2005, ApJ, 623, 314

Preece, R. D., Briggs, M. S., Mallozzi, R. S., et al. 2000, ApJS, 126, 19

Price, P. A., Cowie, L. L., Minezaki, T., et al. 2006, ApJ, 645, 851

Porciani, C., \& Madau, P. 2001, ApJ, 548, 522

Rees, M. J., \& Mészáros, P. 1994, ApJ, 430, L93

Reichart, D. E. 2005, GCN Circ., 3915

Romano, P., Moretti, A., Banat, P. L., et al. 2006, A\&A, 450, 59

Roming, P. W. A., Kennedy, T. E., Mason, K. O., et al. 2005, Space Sci. Rev., 120,95

Sakamoto, T., Barbier, L., Barthelmy, S., et al. 2005, GCN Circ., 3938

Tagliaferri, G., Goad, M., Chincarini, G., et al. 2005a, Nature, 436, 985

Tagliaferri, G., Antonelli, L. A., Chincarini, G., et al. 2005b, A\&A, 443, L1

van Paradijs, J., Groot, P. J., Galama, T., et al. 1997, Nature, 386, 686

Vaughan, S., Goad, M. R., Beardmore, A. P., et al. 2006a, ApJ, 638, 920

Vaughan, S., Willingale, R., Romano, P., et al. 2006b, ApJ, 639, 323

Zhang, B., Fan, Y. Z., Dyks, J., et al. 2005, ApJ, 642, 354

Zou, Y. C., Xu, D., \& Dai, Z. G. 2006, ApJ, 646, 1098

Watson, D., Reeves, J. N., Hjorth, J., et al. 2006, ApJ, 637, L69

Wei, D. M., Yan, T., \& Fan, Y. Z. 2006, ApJ, 636, L69

Woosley, S. E., \& Heher, A. 2006, ApJ, 637, 914 